\title{
Martin Günsche
}

rau F. M., geboren 1960, wurde erstmals im Februar 2004 bei mir vorstellig.

\section{Diagnosen}

- Manisch-depressive Erkrankung

- Konzentrationsstörungen in erheblichem Ausmass

- Therapieresistente Tagesmüdigkeit und Antriebsschwäche

- Status nach Uterusextirpation wegen uterus myomatosus 2003

- Gewichtsprobleme seit 2003

\section{Therapie \\ - Lithiofor \\ - Deroxat \\ - PADMA 28}

Bei der ersten Konsultation beklagte Frau F. M. eine für sie sehr belastende Gewichtszunahme um $15 \mathrm{~kg}$, die seit der Hysterektomie im Juni 2003 bestand. Ausserdem litt sie immer wieder unter einer massiv eingeschränkten Leistungsfähigkeit durch grosse Tagesmüdigkeit und Konzentrationsstörungen. Wegen dieser Beschwerden wurden bereits von vorbehandelnden Ärzten abklärende Untersuchungen durchgeführt, durch die organische Ursachen ausgeschlossen werden

*PADMED CIRCOSAN steht speziell dem Arzt zur Verfügung. Es ist kassenzulässig und identisch mit PADMA 28.

\section{Therapieresistenz bei Tages- müdigkeit, Antriebsschwäche und Konzentrationsstörung}

\author{
Erfolgreiche Behandlung mit PADMA 28*
}

konnten. Die Patientin nimmt bei bestehender manisch-depressiver Erkrankung seit Jahren als Dauermedikation Lithiofor und Deroxat ein. Manische Phasen waren seit vielen Jahren nicht mehr aufgetreten; dagegen kam es in den letzten Jahren noch gelegentlich $\mathrm{zu}$ depressiven Verstimmungen.

Die Gewichtsprobleme konnten unter entsprechender diätetischer Anleitung erfolgreich angegangen werden, so dass sich das Gewicht bis August 2004 um $9 \mathrm{~kg}$ reduzieren liess, was seither auch stabil blieb.

Als therapieresistent hatten sich allerdings die seit Jahren bestehenden Phasen grosser Tagesmüdigkeit und Konzentrationsstörungen erwiesen. Solche Phasen traten nach Angaben der Patientin regelmässig alle 4-6 Wochen auf, seien aber keinesfalls mit depressiven Gedanken oder Ideen verknüpft. Vielmehr fühle sie sich wie in Nebel eingehüllt, so dass sie sich sehr zusammen nehmen müsse, um ihre Tagesgeschäfte - eingeschränkt - erledigen zu können. Die Phasen hielten jeweils 4-8 Tage an und hätten bisher mit nichts beeinflusst werden können. Alle in den letzten Jahren erfolgten internistischen Abklärungen dieser Problematik hätten keine Ursache zu Tage gebracht, was eine gezielte Therapie verunmöglichte.

Wegen dieser Beschwerden wurde von mir als probatorische Massnahme neben oben beschriebenen Psychopharmaka ab Ende Oktober 2004 eine zusätzliche Behandlung mit PADMA 28 2-2-2-0 Tabletten begonnen.

\section{Beschwerden massiv nachgelassen ...}

2 Wochen nach Beginn der Tabletteneinnahme berichtete die Patientin hocherfreut über ein massives Nachlassen von Spannungsgefühlen in Beinen und Brüsten; Beschwerden, die bis anhin zwar nie erwähnt wurden, die Patientin aber bereits seit vielen Jahren belasteten.

6 Wochen nach Therapiebeginn berichtete Frau F. M., dass seit der Einnahme von PADMA 28 keine Extremphasen der Tagesmüdigkeit und Konzentrationsstörungen mehr aufgetreten seien. Sie fühle sich im Gegenteil ganz ausgezeichnet und denke sogar über den Besuch eines Weiterbildungsseminars nach, was ihr zuvor völlig undenkbar erschien. Anlässlich dieses Therapieerfolgs wurde die Dosis von PADMA 28 auf 1-1-1-0 Tabletten reduziert.

\section{... und nachhaltig gebessert}

Ich habe die Patientin zuletzt Ende Januar 2005 gesehen. Sie berichtete über weiterhin nachhaltig gebesserte Konzentrationsfähigkeit und Wachheit bei deutlich gesteigerter intellektueller Leistungsfähigkeit.

Die Patientin ist, ebenso wie ich, mit dem durch PADMA 28 erzielten Therapieergebnis sehr zufrieden.

\section{Anschrift des Autors:}

Dr. med. Martin Günsche Praktischer Arzt FMH Alpenblick, $\mathrm{CH}-8784$ Braunwald 